\title{
Comparison of clinical outcomes with orodispersible versus standard oral olanzapine tablets in nonadherent patients with schizophrenia or bipolar disorder
}

\author{
This article was published in the following Dove Press journal: \\ Patient Preference and Adherence \\ 6 June 2017 \\ Number of times this article has been viewed
}

\section{Diego Novick' \\ William Montgomery ${ }^{2}$ \\ Tamas Treuer ${ }^{3}$ \\ Ai Koyanagi ${ }^{4}$ \\ Jaume Aguado 4 \\ Susanne Kraemer ${ }^{5}$ \\ Josep Maria Haro ${ }^{4}$}

'Global Patient Outcomes and Real World Evidence (GPORWE), Eli Lilly and Company, Windlesham, Surrey, UK; ${ }^{2}$ Global Patient Outcomes and Real World Evidence (GPORWE), Eli Lilly Australia Pty Ltd, West Ryde, Australia; ${ }^{3} \mathrm{Global}$ Patient Outcomes and Real World Evidence (GPORWE), Eli Lilly and Company, Budapest, Hungary; ${ }^{4}$ Parc Sanitari Sant Joan de Déu, CIBERSAM, Universitat de Barcelona, Barcelona, Spain; ${ }^{5}$ Medical Department, Eli Lilly and Company, Bad Homburg, Germany
Correspondence: Diego Novick Global Health Outcomes Research, Eli Lilly and Company, Lilly Research Centre, Erl Wood Manor, Windlesham, Surrey GU20 6PH, UK

Tel +44 I 276483832

Fax +44 I27 6483192

Email Novick_diego@lilly.com
Purpose: Medication nonadherence is common in the treatment of patients with severe mental illness and is a frequent cause of relapse. Different formulations have been developed in an effort to improve medication adherence. The aim of this study was to explore whether there are differential clinical outcomes between two different formulations of olanzapine (orodispersible tablets [ODTs] vs standard oral tablets [SOT]) for the treatment of nonadherent patients with schizophrenia or bipolar disorder.

Methods: Data for this analysis were from an observational study conducted in Europe ( $\mathrm{N}=903)$. Adult schizophrenia and bipolar disorder patients in outpatient settings who initiated or changed to either olanzapine ODT or SOT according to physician decision within the last 45 days were eligible for enrollment. The follow-up period was 1 year. Of the 903 participants, 266 nonadherent patients (Medication Adherence Rating Scale score 0-4 at baseline) were included in the analysis. Clinical outcomes of interest were: 1) hospitalization and 2) relapse identified by the participating psychiatrist or hospitalization. An adjusted logistic regression model was fitted.

Results: Patients taking ODT had more severe illness at baseline $(P<0.001)$ as assessed with the Clinical Global Impression with mean (standard deviation [SD]) scores of ODT 4.63 (1.03) and SOT 4 (1.16). In the regression models adjusted for potential confounders, patients taking ODT had significantly lower odds for hospitalization (odds ratio $=0.355 ; 95 \%$ confidence interval $=0.13-0.974$ ) and relapse or hospitalization (odds ratio $=0.368 ; 95 \%$ confidence inter$\mathrm{val}=0.183-0.739)$, respectively.

Conclusion: Nonadherent patients with schizophrenia or bipolar disorder treated with the orodispersible formulation were less likely to be hospitalized or suffer relapse compared to those patients taking the standard oral coated tablets.

Keywords: olanzapine, schizophrenia, bipolar disorder, orodispersible formulation, relapse, hospitalization

\section{Introduction}

A substantial proportion of patients with severe mental illnesses such as schizophrenia and bipolar disorder do not fully benefit from the wide availability of effective drugs to treat and prevent the symptoms of these conditions due to nonadherence. Although the adherence rate varies widely between studies, it is generally known to be about $40 \%-60 \%$ in patients with schizophrenia, ${ }^{1}$ while $\sim 40 \%$ and $75 \%$ of patients have been reported to stop taking medication within 1 and 2 years, respectively. ${ }^{2}$ Furthermore, a high rate of suboptimal adherence has also been reported in patients with bipolar 
disorder $(69 \%))^{3}$ In a recent systematic review, positive attitudes to medication and illness insight were the only factors consistently associated with better adherence in patients with schizophrenia, while mixed results were found for other factors such as symptom severity and side effects. ${ }^{1}$

Nonadherence in severe mental illness may exacerbate symptoms and lead to relapse and hospitalization. ${ }^{3,4}$ For instance, in patients with a first episode of psychosis, nonadherence was identified as the strongest single predictor of relapse, ${ }^{5}$ while a recent study from China showed that nonadherent patients were 2.5 times more likely to relapse in the year following discharge than adherent patients. ${ }^{6}$ Furthermore, compared to patients with no gaps in medication therapy, those patients with gaps of $>30$ days over a 1 -year period had a nearly fourfold risk of being hospitalized, with even a gap of 1-10 days doubling the risk. ${ }^{7}$

Given that nonadherence is a potentially preventable cause of adverse clinical outcomes including relapse and hospitalization, different types of formulations have been developed in an attempt to improve adherence. These delivery systems include long-acting injections, liquid formulations, oral granules, transdermal patches, and orodispersible tablets (ODTs) ${ }^{8}$ For example, olanzapine is a well-established drug used to treat patients with schizophrenia and bipolar disorder. Oral olanzapine is available in the form of ODTs and standard oral tablets (SOT). The ODT formulation of olanzapine is designed to dissolve upon contact with saliva and cannot be easily spat out, ${ }^{8}$ and may be preferable for patients who are reluctant or unable to swallow tablets. ${ }^{9}$ Previous studies have shown that the ODT formulation is associated with higher adherence rates,${ }^{8,9}$ increased patient preference, ${ }^{10,11}$ and improved ease of administration, which may reduce the burden of treatment not only on patients but also on caregivers. ${ }^{12}$

Despite the potential benefits associated with ODT, ${ }^{13}$ particularly in terms of adherence, there are no studies to date which have compared the effects of different oral forms of olanzapine on the hospitalization and relapse rates in patients with severe mental disorders, while studies on the use and effectiveness of different oral formulations of olanzapine in natural settings (eg, routine care in outpatient settings) are scarce. Furthermore, data on nonadherent patients are limited as they are rarely included in clinical trials. ${ }^{14}$ Thus, the aim of this study was to assess whether nonadherent patients with schizophrenia or bipolar disorder have different hospitalization and relapse rates when treated with ODT compared to SOT within a follow-up period of 12 months in the outpatient settings of three European countries (France, Germany, and Greece).

\section{Methods \\ Study design}

Details regarding the study design have been published previously. ${ }^{15-17}$ Briefly, data for this post hoc analysis were obtained from a prospective, observational (noninterventional), naturalistic, multicenter, multicountry study (France, Germany, and Greece) designed to compare medication adherence between two oral forms of olanzapine (ODT vs SOT) in patients with schizophrenia or bipolar disorder. Patients were followed up for 1 year, with up to five study visits at $\sim 3$-month ( \pm 1 month) intervals. Data collection occurred when the patients attended their regular clinic visits. The participating psychiatrists or their designees conducted the assessments. All investigators attended a start-up meeting which consisted of training in the study procedures and questionnaire administration. Each participating psychiatrist was asked to enroll, consecutively, up to eight eligible patients, in order to limit selection bias.

\section{Patients}

Patients were eligible for enrollment if they met both of the following inclusion criteria: 1 ) adult patients diagnosed with schizophrenia or any type of bipolar disorder based on the Diagnosis and Statistical Manual of Mental Disorders, Fourth Edition, and 2) patients for whom their physician decided to begin antipsychotic treatment with olanzapine (either ODT or SOT), according to the approved marketing authorization, within the last 45 days (either as treatment initiation or as switch from another antipsychotic). The exclusion criteria were the following: 1) currently receiving treatment with investigational drugs or procedures at enrollment (or during the study period) and 2) any contraindication for olanzapine (eg, hypersensitivity, risk for narrow angle glaucoma).

A total of 903 patients with schizophrenia or any type of bipolar disorder from outpatient or hospital settings were enrolled in this study between April 2007 and April 2008. The last study visit occurred in May 2009. Patients with schizoaffective disorder were not included in the analysis, as olanzapine is not approved specifically for its treatment in the countries included in the study. The protocol did not restrict use to antipsychotic monotherapy or combination therapy, in order to obtain an unbiased sample. This was also done to reflect medication decisions in real-world settings where combination therapy is not uncommon. All treatment decisions were made at the discretion of the treating physician and patient, including the choice of olanzapine formulation. Of the 903 patients, 266 nonadherent patients, defined as having a baseline rating of $0-4$ in the Medication Adherence 
Rating Scale (MARS), constituted the final analytical sample (details of this scale can be found below). Although the MARS does not have an established cut-off, in line with a previous study using the same dataset, ${ }^{16}$ we used 4 as the cutoff for MARS. This corresponded to the lowest tertile of the MARS score distribution, and we judged that scores in this range are highly likely to have clinical implications.

\section{Ethical approval}

The study was approved by ethical review boards as required by local law and was conducted in accordance with the ethical principles that have their origin in the Declaration of Helsinki. The specific ethics committees that approved the study are: 1) Comité consultatif sur le traitement de l'information en matière de recherche dans le domaine de la santé (France), 2) Landesärztekammer Hessen EthikKommission (Germany), and 3) Papageorgiou Regional General Hospital of Thessaloniki (Greece). All patients provided written informed consent.

\section{Outcome}

Two clinical outcomes during the follow-up period (12 months) were assessed in this analysis: 1) inpatient hospitalization for any reason and 2) relapse. Relapse was defined as either an inpatient hospitalization or a relapse identified by the participating psychiatrist. Information on these events was recorded during follow-up at each visit.

\section{Other variables}

Clinical severity was measured with the Clinical Global Impression (CGI) scale for Bipolar Disorder (CGI-BP) ${ }^{18}$ or Schizophrenia (CGI-SCH). ${ }^{19}$ However, for this study, we only used the general severity score, which is included in both scales and is equivalent to the original CGI score, for the sake of consistency. Medication adherence was measured with the MARS, which is a ten-item self-reported measure ranging from 0 to 10 , with higher scores representing better adherence. The MARS has demonstrated a high level of validity compared to existing self-report measures, and assesses a range of behaviors and attitudes linked to adherence. ${ }^{20}$ Information on physical comorbidities such as diabetes, hypertension, hyperlipidemia, and obesity was also obtained. Those who had at least one physical comorbidity were considered to have any physical condition.

\section{Statistical analysis}

Baseline patient characteristics and by treatment and by relapse were described and compared using the chi-squared test (or Fischer's exact test) and Mann-Whitney $U$ tests for categorical and continuous variables, respectively. To study the effect of the baseline treatment form on the post-baseline hospitalization as well as the relapse rates, multivariable logistic regression models were employed. The models were adjusted for relevant baseline covariates (CGI, diagnosis, sex, country, age, any physical condition, and time since first episode). CGI, age, and time since first episode were included in the models as continuous variables, whereas other variables were included as categorical variables. Odds ratios (ORs) and 95\% confidence intervals (CIs) are presented. A $P$-value $<0.05$ was considered to be statistically significant. Data analysis was carried out with $\mathrm{SAS}^{\circledR}$ software (SAS Institute, Cary, NC, USA), version 9.3.

\section{Results}

A total of 266 nonadherent patients (116 females and 150 males) at baseline were included in the analysis, of whom 195 and 71 had schizophrenia and bipolar disorder, respectively. The mean age (standard deviation [SD]) was 38.7 (12.6) years. SOT and ODT were administered to 89 and 177 patients, respectively. Of these 266 individuals, at the final visit, only $33.7 \%$ continued to be nonadherent, and there were 8 individuals who had to discontinue the study for incomplete compliance ( $n=3$; SOT 2 vs ODT 1), patient request ( $\mathrm{n}=4$; SOT 3 vs ODT 1$)$, and intolerability $(\mathrm{n}=1$; ODT 1). The mean (SD) MARS scores at the final visit were not significantly different between the two treatment groups (SOT 5.5 [2.6] and ODT 5.9 [2.5]; Student's $t$-test $P=0.2994)$.

There was a significant difference in terms of the proportion of those taking different forms of tablets by country; ODTs were used more than twice as frequently in Greece as in France (Table 1). Furthermore, the mean CGI was significantly higher (ie, higher disease severity) among those taking ODT compared to those taking SOT (4.63 vs 4; $P<0.001)$. There were no significant differences in terms of sex, diagnosis, any physical condition, age, years since first treatment, and MARS score between the two treatment groups. The relapse rates by the baseline characteristics are provided in Table 2. Patients who experienced relapse during the follow-up period were significantly more likely to have at least one physical health condition, longer time since first treatment, and higher CGI scores at baseline.

The overall hospitalization and relapse rates were $9.8 \%$ and $28.6 \%$, respectively. In the unadjusted analysis, the hospitalization rate was significantly higher in the SOT group compared to the ODT group ( $16.9 \%$ vs $6.2 \% ; P=0.0058)$. 
Table I Patient baseline demographic and clinical characteristics by treatment form

\begin{tabular}{|c|c|c|c|c|}
\hline Variables & $\begin{array}{l}\text { Overall }^{a} \\
(\mathrm{~N}=266)\end{array}$ & $\begin{array}{l}\text { SOT }^{\mathrm{b}} \\
(\mathrm{n}=89)\end{array}$ & $\begin{array}{l}\text { ODT }^{\mathrm{b}} \\
(\mathrm{n}=177)\end{array}$ & $P$-value \\
\hline Sex & & & & 0.5664 \\
\hline Female & $43.6 \%$ & $35.3 \%$ & $64.7 \%$ & \\
\hline Male & $56.4 \%$ & $32 \%$ & $68 \%$ & \\
\hline Country & & & & $<0.0001$ \\
\hline France & $28.2 \%$ & $62.7 \%$ & $37.3 \%$ & \\
\hline Germany & $22.9 \%$ & $39.3 \%$ & $60.7 \%$ & \\
\hline Greece & $48.9 \%$ & $13.8 \%$ & $86.2 \%$ & \\
\hline Diagnosis & & & & 0.9428 \\
\hline Schizophrenia & $73.3 \%$ & $33.3 \%$ & $66.7 \%$ & \\
\hline Bipolar disorder & $26.7 \%$ & $33.8 \%$ & $66.2 \%$ & \\
\hline Any physical condition(s) ${ }^{d}$ & & & & 0.7432 \\
\hline No & $74.8 \%$ & $32.7 \%$ & $67.3 \%$ & \\
\hline Yes & $25.2 \%$ & $34.9 \%$ & $65.1 \%$ & \\
\hline Age (years); mean (SD) & $38.7(12.6)$ & $40.2(13.5)$ & $38(12)$ & 0.2188 \\
\hline Years since first treatment; mean (SD) ${ }^{\mathrm{e}}$ & I2.I (9.7) & I3.5 (I0.7) & I I.4 (9.I) & 0.1209 \\
\hline MARS at study entryf; mean (SD) & $2.5(1.37)$ & $2.45(1.31)$ & $2.53(1.40)$ & 0.5494 \\
\hline CGI; mean (SD) & $4.42(1.11)$ & $4(1.16)$ & $4.63(1.03)$ & $<0.0001$ \\
\hline \multicolumn{5}{|c|}{ Polypharmacy (in conjunction with olanzapine) } \\
\hline No & $83.5 \%$ & $33.8 \%$ & $66.2 \%$ & 0.8007 \\
\hline Yes & $16.5 \%$ & $31.8 \%$ & $68.2 \%$ & \\
\hline \multicolumn{5}{|l|}{ Antipsychotics taken prior to study entry } \\
\hline Amisulpride & $8.3 \%$ & $18.2 \%$ & $81.8 \%$ & 0.1128 \\
\hline Aripiprazole & $3.8 \%$ & $40 \%$ & $60 \%$ & $0.7359^{9}$ \\
\hline Chlorpromazine & $7.1 \%$ & $31.6 \%$ & $68.4 \%$ & 0.8570 \\
\hline Clozapine & $2.6 \%$ & $57.1 \%$ & $42.9 \%$ & $0.2278^{8}$ \\
\hline Fluphenazine & $3.4 \%$ & $11.1 \%$ & $88.9 \%$ & $0.2797^{8}$ \\
\hline Haloperidol & $29.7 \%$ & $24.1 \%$ & $75.9 \%$ & 0.0345 \\
\hline Perphenazine & $3.4 \%$ & $44.4 \%$ & $55.6 \%$ & $\left.0.488\right|^{8}$ \\
\hline Quetiapine & $4.9 \%$ & $23.1 \%$ & $76.9 \%$ & $0.5532^{g}$ \\
\hline Risperidone & $22.6 \%$ & $28.3 \%$ & $71.7 \%$ & 0.3390 \\
\hline Ziprasidone & $4.9 \%$ & $7.7 \%$ & $92.3 \%$ & $0.0664^{g}$ \\
\hline Zuclopenthixol & $2.6 \%$ & $57.1 \%$ & $42.9 \%$ & $0.2278^{g}$ \\
\hline Other antipsychotic & $4.5 \%$ & $33.3 \%$ & $66.7 \%$ & $1.000^{\mathrm{g}}$ \\
\hline
\end{tabular}

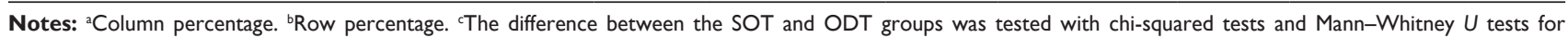
categorical and continuous variables, respectively. ${ }^{\mathrm{d}} \mathrm{n}=262$ (overall), $\mathrm{n}=87$ (SOT), $\mathrm{n}=175$ (ODT). ${ }^{\mathrm{n}} \mathrm{n}=258$ (overall), $\mathrm{n}=87$ (SOT), $\mathrm{n}=$ I7I (ODT). ${ }^{\mathrm{T}}$ The MARS ranges from 0 to I0,

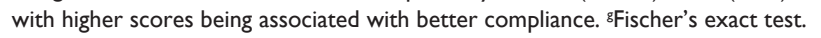

Abbreviations: CGI, Clinical Global Impression; MARS, Medication Adherence Rating Scale; ODT, orodispersible tablet; SD, standard deviation; SOT, standard oral tablets.

Although the relapse rate was also higher among patients on SOT compared to ODT ( $36 \%$ vs $24.9 \%$ ), the difference was of borderline statistical significance $(P=0.059$; Figure 1$)$. The effect of treatment form on hospitalization (Table 3 ) and relapse (Table 4 ) estimated by multivariate logistic regression revealed that patients taking ODT had significantly $(64.5 \%$ and $63.2 \%$, respectively) lower odds for hospitalization $(\mathrm{OR}=0.355 ; 95 \% \mathrm{CI}=0.13-0.974)$ and relapse $(\mathrm{OR}=0.368$; $95 \%$ CI $=0.183-0.739)$, respectively.

Our study protocol included individuals who were on polypharmacy (ie, taking another antipsychotic in conjunction with olanzapine). In order to assess whether this affected our results, we conducted a sensitivity analysis by excluding those who were on polypharmacy from the analysis. The sensitivity analysis showed that there were no appreciable changes in the results, meaning that polypharmacy was unlikely to have affected our results.

\section{Discussion}

This post hoc analysis examined the clinical outcomes of nonadherent schizophrenia and bipolar disorder patients treated with either SOT or ODT at baseline over a 1-year period. The study found that patients on ODT had $64.5 \%-63.2 \%$ lower odds for hospitalization and/or relapse compared to those on SOT, despite the fact that disease severity was more pronounced in the ODT group at baseline. Our study results 
Table 2 Baseline demographics and clinical characteristics of patients by relapse

\begin{tabular}{|c|c|c|c|}
\hline \multirow[t]{2}{*}{ Variables } & \multicolumn{3}{|l|}{ Relapse } \\
\hline & No & Yes & $P$-value ${ }^{a}$ \\
\hline \multicolumn{4}{|l|}{ Sex (\%) } \\
\hline Female & 70.7 & 29.3 & 0.8145 \\
\hline Male & 72.0 & 28.0 & \\
\hline \multicolumn{4}{|l|}{ Country (\%) } \\
\hline France & 65.3 & 34.7 & 0.09 \\
\hline Germany & 82 & 18 & \\
\hline Greece & 70 & 30 & \\
\hline \multicolumn{4}{|l|}{ Diagnosis (\%) } \\
\hline Schizophrenia & 73.3 & 26.7 & 0.2544 \\
\hline Bipolar disorder & 66.2 & 33.8 & \\
\hline \multicolumn{4}{|l|}{ Any physical condition (\%) } \\
\hline No & 7 & 25 & 0.0252 \\
\hline Yes & 60.6 & 39.4 & \\
\hline Age (years); mean (SD) & $38(12.4)$ & $40.6(12.9)$ & 0.1035 \\
\hline $\begin{array}{l}\text { Years since first treatment; } \\
\text { mean }(S D)\end{array}$ & II.I (9.4) & $14.5(10.1)$ & 0.0066 \\
\hline MARS'; mean (SD) & $2.58(1.34)$ & $2.30(1.42)$ & 0.1592 \\
\hline CGI; mean (SD) & $4.32(1.05)$ & $4.66(1.24)$ & 0.0051 \\
\hline
\end{tabular}

Notes: ${ }^{a}$ The difference between the relapse groups was tested with chi-squared tests and Mann-Whitney $U$ tests for categorical and continuous variables, respectively. 'The MARS ranges from 0 to 10 , with higher scores being associated with better compliance.

Abbreviations: CGI, Clinical Global Impression; MARS, Medication Adherence Rating Scale; SD, standard deviation.

point to a possible clinical benefit of ODT in nonadherent patients - a group for which only limited data exist due to their tendency to not participate in clinical trials.

Our finding that those on ODT had more severe disease at baseline is in line with a previous study ${ }^{9}$ and may reflect attitudes of psychiatrists who tend to prescribe ODT to patients who are more likely to be noncompliant, severely ill, or aggressive. The lower hospitalization/relapse rate among patients on ODT, despite more severe illness at baseline, may be attributable to improvements in adherence, given that nonadherence has been reported to be the single strongest predictor of relapse. ${ }^{5}$

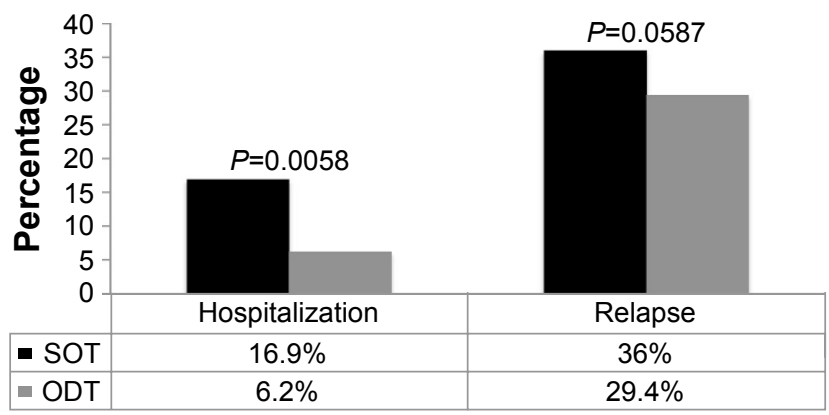

Figure I Hospitalization and relapse rate by medication formulation at baseline. Abbreviations: ODT, orodispersible tablet; SOT, standard oral tablets.
Table 3 Effect of treatment form on hospitalization estimated by multivariable logistic regression

\begin{tabular}{lllll}
\hline Effect & OR & $\begin{array}{l}\text { 95\% Wald } \\
\text { confidence } \\
\text { limits }\end{array}$ & P-value \\
\hline ODT (vs SOT) & 0.355 & 0.13 & 0.974 & 0.0444 \\
CGI & 1.622 & 1.045 & 2.516 & 0.0309 \\
Schizophrenia & 1.074 & 0.387 & 2.984 & 0.8907 \\
(vs bipolar disorder) & & & & \\
Female (vs male) & 1.295 & 0.522 & 3.216 & 0.5773 \\
Country & & & & 0.0535 \\
$\quad$ France & 1 & 1 & 1 & \\
$\quad$ Germany & 0.247 & 0.063 & 0.974 & \\
$\quad$ Greece & 0.35 & 0.119 & 1.025 & \\
Age (years) & 0.974 & 0.918 & 1.034 & 0.3867 \\
Any physical conditions & 1.203 & 0.423 & 3.42 & 0.7289 \\
Time since first episode (years) & 1.039 & 0.97 & 1.114 & 0.2762 \\
\hline
\end{tabular}

Note: Model is adjusted for all variables in the table.

Abbreviations: CGI, Clinical Global Impression; ODT, orodispersible tablet; OR, odds ratio; SOT, standard oral tablets.

In an observational study with a follow-up period of 6 weeks, ODT was not only significantly associated with improvements in clinical symptoms, but also with improvements in adherence, attitude, and nursing care burden in acutely ill nonadherent patients with schizophrenia. ${ }^{8}$ In particular, improvements in adherence were observed at as early as 1 week. Also in this study, although the sample consisted of noncompliant patients at baseline, the majority (75.3\%) of patients completed the 6-week study and expressed positive opinions about taking ODT throughout the study period. It has been suggested that ODT tablets may increase acceptance of medication due to ease of intake and the difficulty in discarding the dose, and the evident improvements in psychotic symptoms accompanied by minimal side effects.

Table 4 Effect of treatment form on relapse estimated by multivariable logistic regression

\begin{tabular}{lllll}
\hline Effect & OR & $\begin{array}{l}\text { 95\% Wald } \\
\text { confidence } \\
\text { limits }\end{array}$ & P-value \\
\hline ODT (vs SOT) & 0.368 & 0.183 & 0.739 & 0.0049 \\
CGI & 1.545 & 1.158 & 2.06 & 0.0031 \\
Schizophrenia & 0.809 & 0.415 & 1.579 & 0.5347 \\
(vs bipolar disorder) & & & & \\
Female (vs male) & 1.068 & 0.58 & 1.969 & 0.8317 \\
Country & & & & \\
$\quad$ France & 1 & 1 & 1 & 0.1396 \\
$\quad$ Germany & 0.501 & 0.206 & 1.222 & \\
$\quad$ Greece & 1.158 & 0.551 & 2.433 & \\
Age (years) & 0.99 & 0.956 & 1.026 & 0.5884 \\
Any physical conditions & 1.756 & 0.891 & 3.46 & 0.1036 \\
Time since first episode (years) & 1.034 & 0.99 & 1.081 & 0.1334 \\
\hline Note: Modelis adyus
\end{tabular}

Note: Model is adjusted for all variables in the table.

Abbreviations: CGI, Clinical Global Impression; ODT, orodispersible tablets; OR, odds ratio; SOT, standard oral tablets. 
Furthermore, in another observational study of acutely ill patients with schizophrenia requiring emergency treatment, increase in the rate of medication acceptance was more pronounced in the ODT group compared to the SOT group as follows: (baseline vs after 2 weeks) positive attitude toward medication (ODT $31.6 \%$ vs $68.4 \%$; SOT $58.0 \%$ vs $78.3 \%$ ), ingestion (ODT $48.9 \%$ vs $83.4 \%$; SOT $75.4 \%$ vs $89.4 \%$ ), and nursing effort (ie, no extensive nursing effort was needed to administer medication; ODT $53.9 \%$ vs $86.2 \%$; SOT $81.1 \%$ vs $92.8 \%){ }^{9}$

In addition, it is of particular importance to consider the patient's preference in terms of treatment in disorders such as schizophrenia and bipolar disorder, which frequently require long-term treatment, as this may influence longer-term adherence with treatment and subsequent clinical outcomes. In a 12-week randomized, crossover, open-label study, patients with stable schizophrenia who were on olanzapine SOT monotherapy were randomly assigned to ODT or SOT to assess patient preference for these different formulations. At the end of the study, a significantly higher proportion of patients claimed to prefer ODT over SOT (61\% vs 27\%; $P<0.001) .{ }^{11}$

The study results should be interpreted in light of several limitations. First, we were unable to conduct stratified analyses by disorder type (ie, schizophrenia or bipolar disorder) due to the small sample size. Second, the data were drawn from an observational study in which allocation of treatment form was not at random. While we adjusted for potential confounders, it is possible that there are other factors related to clinical outcome and physician's choice of drug formulation that were not measured in the study. Thus, our results should not be considered as a direct comparison of the effects of the two different formulations of olanzapine. Third, medication adherence is notoriously difficult to measure, as the simple act of measuring adherence via selfadministered questionnaires, physician assessment, or pill counts may influence patient behavior. ${ }^{21}$ Despite this, the fact that our results were based on routine care provides us with the unique opportunity to observe the effectiveness of treatments in natural settings, which may be different from studies conducted under experimental conditions, as in a clinical trial. Fourth, the selection of the patients included in the study was based on the MARS, which measures general attitudes toward medication. Although $82 \%$ of the patients included in the analysis were taking antipsychotics immediately before study entry, the rest may have based their answers for the MARS on experiences with other types of medication. Finally, assessment was not blind. However, this was a post hoc analysis in which the investigators were not aware of the study question.

\section{Conclusion}

This analysis from a real-world study found that in nonadherent patients with schizophrenia or bipolar disorder, those patients treated with the orodispersible formulation were less likely to be hospitalized and/or suffer relapse compared to those treated with the standard oral coated tablets, in spite of having more severe disease at baseline. More research is needed to uncover the factors which may underlie our findings. Specifically, features of ODT such as ease of use, patient preference, and better adherence may be responsible for our findings, and these warrant further investigation.

\section{Acknowledgment}

This study was funded by Eli Lilly and Co.

\section{Disclosure}

Diego Novick, William Montgomery, Tamas Treuer, and Susanne Kraemer are Lilly employees. Jaume Aguado conducted the statistical analyses under a contract of CIBERSAM with Lilly. Josep Maria Haro has received personal fees from Eli Lilly and Co., Roche, Lundbeck, and Otsuka. The authors report no other conflicts of interest in this work.

\section{References}

1. Sendt KV, Tracy DK, Bhattacharyya S. A systematic review of factors influencing adherence to antipsychotic medication in schizophreniaspectrum disorders. Psychiatry Res. 2015;225(1-2):14-30.

2. Perkins DO. Adherence to antipsychotic medications. JClin Psychiatry. 1999;60(Suppl 21):25-30.

3. Montes JM, Maurino J, de Dios C, Medina E. Suboptimal treatment adherence in bipolar disorder: impact on clinical outcomes and functioning. Patient Prefer Adherence. 2013;7:89-94.

4. American Psychiatric Association. Practive Guidelines for the Treatment of Patients with Schizophrenia. 2nd ed. Washington, DC: American Psychiatric Association; 2004.

5. Caseiro O, Perez-Iglesias R, Mata I, et al. Predicting relapse after a first episode of non-affective psychosis: a three-year follow-up study. J Psychiatr Res. 2012;46(8):1099-1105.

6. Xiao J, Mi W, Li L, Shi Y, Zhang H. High relapse rate and poor medication adherence in the Chinese population with schizophrenia: results from an observational survey in the People's Republic of China. Neuropsychiatr Dis Treat. 2015;11:1161-1167.

7. Weiden PJ, Kozma C, Grogg A, Locklear J. Partial compliance and risk of rehospitalization among California Medicaid patients with schizophrenia. Psychiatr Serv. 2004;55(8):886-891.

8. Kinon BJ, Hill AL, Liu H, Kollack-Walker S. Olanzapine orally disintegrating tablets in the treatment of acutely ill non-compliant patients with schizophrenia. Int J Neuropsychopharmacol. 2003;6(2):97-102.

9. Czekalla J, Wagner T, Schacht A, Kluge M, Kinon B. Effectiveness and medication acceptance of olanzapine disintegrating tablets compared to standard olanzapine tablets in acutely treated psychiatric patients. Patient Prefer Adherence. 2007;1:19-27.

10. Chue P, Jones B, Taylor CC, Dickson R. Dissolution profile, tolerability, and acceptability of the orally disintegrating olanzapine tablet in patients with schizophrenia. Can J Psychiatry. 2002;47(8):771-774. 
11. Bitter I, Treuer T, Dilbaz N, et al. Patients' preference for olanzapine orodispersible tablet compared with conventional oral tablet in a multinational, randomized, crossover study. World J Biol Psychiatry. 2010;11(7):894-903.

12. San L, Casillas M, Ciudad A, Gilaberte I. Olanzapine orally disintegrating tablet: a review of efficacy and compliance. CNS Neurosci Ther. 2008;14(3):203-214.

13. Montgomery W, Treuer T, Karagianis J, Ascher-Svanum H, Harrison G. Orally disintegrating olanzapine review: effectiveness, patien preference, adherence, and other properties. Patient Prefer Adherence. 2012;6:109-125

14. Barnett PG, Scott JY, Rosenheck RA, Group CSPS. How do clinical trial participants compare to other patients with schizophrenia? Schizophr Res. 2011;130(1-3):34-39.

15. Kraemer S, Chartier F, Augendre-Ferrante B, et al. Effectiveness of two formulations of oral olanzapine in patients with schizophrenia or bipolar disorder in a natural setting: results from a 1-year European observational study. Hum Psychopharmacol. 2012;27(3):284-294.

16. Novick D, Montgomery W, Treuer T, Aguado J, Kraemer S, Haro JM. Comparative effectiveness in terms of treatment discontinuation of orodispersable versus standard oral olanzapine tablets in non-adherent patients: results from a 1-year European Outpatient Observational Study. Value Health. 2014;17(7):A766.
17. Novick D, Montgomery W, Treuer T, Aguado J, Kraemer S, Haro JM Relationship of insight with medication adherence and the impact on outcomes in patients with schizophrenia and bipolar disorder: results from a 1-year European outpatient observational study. BMC Psychiatry. 2015;15:189.

18. Spearing MK, Post RM, Leverich GS, Brandt D, Nolen W. Modification of the Clinical Global Impressions (CGI) Scale for use in bipolar illness (BP): the CGI-BP. Psychiatry Res. 1997;73(3):159-171.

19. Haro JM, Kamath SA, Ochoa S, et al. The Clinical Global ImpressionSchizophrenia scale: a simple instrument to measure the diversity of symptoms present in schizophrenia. Acta Psychiatr Scand Suppl. 2003;(416):16-23.

20. Thompson K, Kulkarni J, Sergejew AA. Reliability and validity of a new Medication Adherence Rating Scale (MARS) for the psychoses. Schizophr Res. 2000;42(3):241-247.

21. Lam WY, Fresco P. Medication adherence measures: an overview. Biomed Res Int. 2015;2015:217047.
Patient Preference and Adherence

\section{Publish your work in this journal}

Patient Preference and Adherence is an international, peer-reviewed, open access journal that focuses on the growing importance of patient preference and adherence throughout the therapeutic continuum. Patient satisfaction, acceptability, quality of life, compliance, persistence and their role in developing new therapeutic modalities and compounds to optimize

\section{Dovepress}

clinical outcomes for existing disease states are major areas of interest for the journal. This journal has been accepted for indexing on PubMed Central. The manuscript management system is completely online and includes a very quick and fair peer-review system, which is all easy to use. Visit http://www. dovepress.com/testimonials.php to read real quotes from published authors. 